\title{
Synthesis and Optical Characterization of Sulfonated Polyaniline/Single-Walled Carbon Nanotube/Zinc Sulphide Nanocomposite
}

\author{
Rajesh K. Agrawalla, ${ }^{1,2}$ Rima Paul, ${ }^{1,3}$ Amit K. Chakraborty, ${ }^{2}$ and Apurba K. Mitra ${ }^{1,4}$ \\ ${ }^{1}$ Nanoscience Laboratory, Department of Physics, National Institute of Technology Durgapur, M.G. Avenue, Durgapur 713209, India \\ ${ }^{2}$ Carbon Nanotechnology Laboratory, Department of Physics, National Institute of Technology Durgapur, \\ M.G. Avenue, Durgapur 713209, India \\ ${ }^{3}$ APC College, New Barrackpore, Kolkata 700131, India \\ ${ }^{4}$ National Institute of Technology-Durgapur, Durgapur 713209, India
}

Correspondence should be addressed to Apurba K. Mitra; akmrecdgp@yahoo.com

Received 5 September 2013; Accepted 28 September 2013

Academic Editors: S. Maksimenko and Z. Shi

Copyright (c) 2013 Rajesh K. Agrawalla et al. This is an open access article distributed under the Creative Commons Attribution License, which permits unrestricted use, distribution, and reproduction in any medium, provided the original work is properly cited.

\begin{abstract}
Polyaniline functionalized with sulfonate groups (SPANI) shows excellent solubility in water. Single-walled carbon nanotubes functionalized with carboxylic groups (f-SWCNTs) and then hybridized with freshly prepared zinc sulphide (ZnS) nanocrystals have been found to be good luminescent material with tuned emission properties. Nanocomposite of sulfonated polyaniline with embedded SWCNT/ZnS nanohybrid fibers has been prepared by a simple solution mixing process and characterized by using high resolution transmission electron microscopy (HRTEM), X-ray diffractometry, Raman spectroscopy, FTIR spectroscopy, and thermogravimetric analysis (TGA). The study of optical properties by UV-vis absorption and photoluminescence spectroscopy reveals that the composite is a luminescent material of enhanced emission intensity in the visible region of the spectrum.
\end{abstract}

\section{Introduction}

Since their discovery by Iijima [1] in 1991, carbon nanotubes (CNTs) have shown remarkable physical properties [2]. Polyaniline (PANI) is the most studied conducting polymer in recent years due to its numerous potential applications. The CNTs have been extensively used as reinforcing fibers in PANI matrix to improve the characteristic optical and electrical properties of the polymer. The PANI/CNT composite finds application in biosensors [3], supercapacitors [4], metal-semiconductor devices [5], and actuators [6]. Zinc sulphide $(\mathrm{ZnS})$ is a wide band gap compound semiconductor and has application as luminescent material $[7,8] . \mathrm{ZnS}$ has been embedded in the matrix of PANI and the resulting PANI-ZnS composite has shown improved optical, electrical, and thermal properties. Dutta et al. [9] dispersed ZnS nanoparticles in PANI matrix and investigated the optical and electrical properties of the hybrid nanostructure. Pant et al.
[10] studied the DC conductivity and dielectric constant of PANI-ZnS composite and found an increase in the values compared to those of pure PANI. Bompilwar et al. [11] made thermogravimetric analysis (TGA) of PANI-ZnS nanocomposite and concluded that the PANI-ZnS composites are thermally more stable than pure PANI. Singh et al. [12] prepared and characterized hybrid composite containing polyaniline, polyethylene glycol, and zinc sulfide. The photoluminescence characteristics of CNT/ZnS hybrid nanostructures have been studied by Paul et al. [13].

While there are published reports on CNT-based SPANI composites [14, 15], as well as ZnS-based PANI composites $[9-12,16]$, to the best of our knowledge, there is no published work to date on composites of SPANI embedded with SWCNT/ZnS hybrid nanofibers. We report here a simple chemical synthesis process of a polymer composite containing SPANI with SWCNT/ZnS nanohybrid reinforcement fibers. SPANI was prepared by sulfonation of emeraldine salt 
of PANI through treatment with chlorosulfonic acid in an inert solvent. This composite is a promising optical material and may find potential applications in sensor and optoelectronic devices.

\section{Experimental}

2.1. Materials. The SWCNTs used in our work were supplied by Chengdu Organic Chemicals Co. Ltd., China, with average diameter, length, and purity, as stated by the manufacturer, being 1-2 nm, 1-3 $\mu \mathrm{m}$, and $95 \mathrm{wt} \%$, respectively. Aniline, 1,2dichloroethane (DCE), and ammonium persulfate were supplied by Merck Specialties Pvt. Ltd., Mumbai. The chlorosulfonic acid was supplied by LOBA Chemie Pvt. Ltd., Mumbai. Except for SWCNTs, all other chemicals were used as received without further purification.

2.2. Purification and Functionalization of SWCNTs. The asreceived SWCNTs were purified by heating in a muffle furnace at $350^{\circ} \mathrm{C}$ in air for $6 \mathrm{~h}$ followed by soaking and stirring in $6 \mathrm{M} \mathrm{HCl}$ for $12 \mathrm{~h}$. The acid-treated SWCNTs were filtered using vacuum filtration system (Millipore, pore size $\sim 0.22 \mu \mathrm{m}$ ) and washed thoroughly with deionized water. The purified SWCNTs were further treated in a mixture of concentrated $\mathrm{HNO}_{3} / \mathrm{H}_{2} \mathrm{SO}_{4}$ in $1: 3$ volume proportion for $4 \mathrm{~h}$ followed by washing with dilute $\mathrm{NaOH}$ aqueous solution and filtration with Millipore filtration apparatus until the $\mathrm{pH}$ became neutral, thereby attaching carboxylic acid $(-\mathrm{COOH})$ groups to obtain the functionalized single-walled carbon nanotubes (f-SWCNT).

2.3. Synthesis of SWCNT/ZnS Hybrid Nanostructures. We took $30 \mathrm{~mL}$ of zinc nitrate solution and 0.0995 gram of $\mathrm{f}$ SWCNT was dispersed in it. The solution was stirred in a magnetic stirrer for $1 \mathrm{~h}$ at room temperature. $15 \mathrm{~mL}$ of methanol with sodium sulfide flakes added to it was sonicated to obtain a saturated solution which was added dropwise to the zinc nitrate solution containing SWCNTs till the $\mathrm{pH}$ became 7. The solution was stirred for $1 \mathrm{~h}$ and filtered using Whatman filter paper. The precipitate was washed with methanol followed by deionized water and filtered again. The sample was then dried under the IR lamp.

2.4. Synthesis of Sulfonated Polyaniline (SPANI). $0.2 \mathrm{M}$ aniline hydrochloride and $0.25 \mathrm{M}$ ammonium persulfate solution were prepared and mixed in equal volumes and left overnight for polymerization to take place. The salt precipitate (PANI) was collected by filtration using a Whatman filter paper. It was then mixed with 1,2-DCE and heated to $80^{\circ} \mathrm{C}$ under stirring using a magnetic stirrer (REMI $2 \mathrm{MLH}$ ). Chlorosulfonic acid diluted with DCE was added dropwise to the reaction mixture and stirred at $80^{\circ} \mathrm{C}$ for $1 \mathrm{~h}$. The semisolid precipitate of sulfonated polyaniline (SPANI) obtained by filtration was then mixed with $400 \mathrm{~mL}$ deionized water and heated to $60^{\circ} \mathrm{C}$ and stirred for $2 \mathrm{~h}$ to promote hydrolysis. The solution was further diluted with water and filtered through a cellulose membrane using a vacuum filtration system

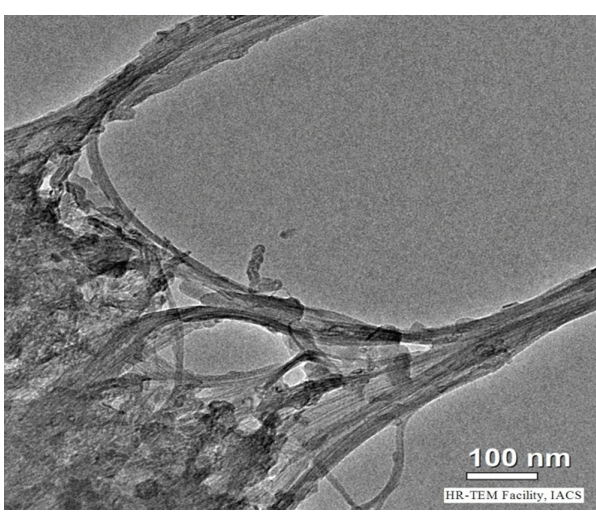

FIGURE 1: HRTEM image of SPANI/SWCNT/ZnS nanocomposite.

(Millipore). The final sample (SPANI) was collected over the filter membrane and dried in air at room temperature.

2.5. Synthesis of SPANI/SWCNT/ZnS Composites. The SWCNT/ZnS hybrid was dispersed in deionized water and ultrasonicated. The prepared dispersion was mixed with SPANI dispersion in deionized water with constant stirring. The resulting solution was heated at $50-60^{\circ} \mathrm{C}$ for $2-3$ hours, with stirring. Then, it was cooled and filtered using vacuum filtration system (Millipore). The sample collected by filtration was dried in air at room temperature to get SPANI/ SWCNT/ZnS composite with SWCNT content of $6 \mathrm{wt} . \%$ and $\mathrm{ZnS}$ content of 7 wt.\%.

2.6. Characterization of the Samples. The micrographs of the sample were obtained using high resolution transmission electron microscope (HRTEM model JEOL JEM-2010; operating acceleration voltage-200 kV). The XRD patterns were obtained using Philips PANalytical X-Pert Pro diffractometer. The molecular structures of the samples were characterized by a Perkin Elmer Spectrum RX I FTIR Spectrometer. The laser source used in the spectrometer was a He-Ne laser $(633 \mathrm{~nm})$. Raman spectroscopy was performed using EZRaman-M field portable Raman analyzer (Enwave Optronics, Inc.). A diode laser of wavelength $785 \mathrm{~nm}$ was used as excitation source. The thermogravimetric analysis (TGA) was carried out with Perkin-Elmer Pyris-1 TGA thermogravimetric analyzer at a heating rate of $10^{\circ} \mathrm{C} / \mathrm{min}$ in nitrogen atmosphere. The optical absorbance spectra were recorded using a HITACHI U-3010 UV-visible absorption spectrophotometer. Photoluminescence spectra of the samples were acquired using a Perkin-Elmer LS-55 fluorescence spectrophotometer.

The scheme of reactions is as in Scheme 1.

\section{Results and Discussion}

\subsection{Structural Characterization}

3.1.1. Microscopy. The HRTEM micrograph of SPANI/ SWCNT/ZnS nanocomposite is shown in Figure 1. We observe that $\mathrm{ZnS}$ nanocrystallites are coated on the surfaces of SWCNT bundles to form SWCNT/ZnS nanohybrids 


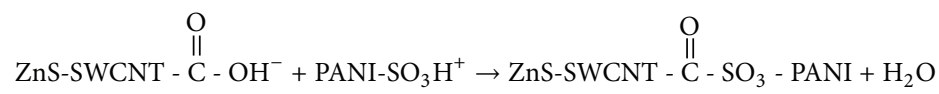

SCHEME 1

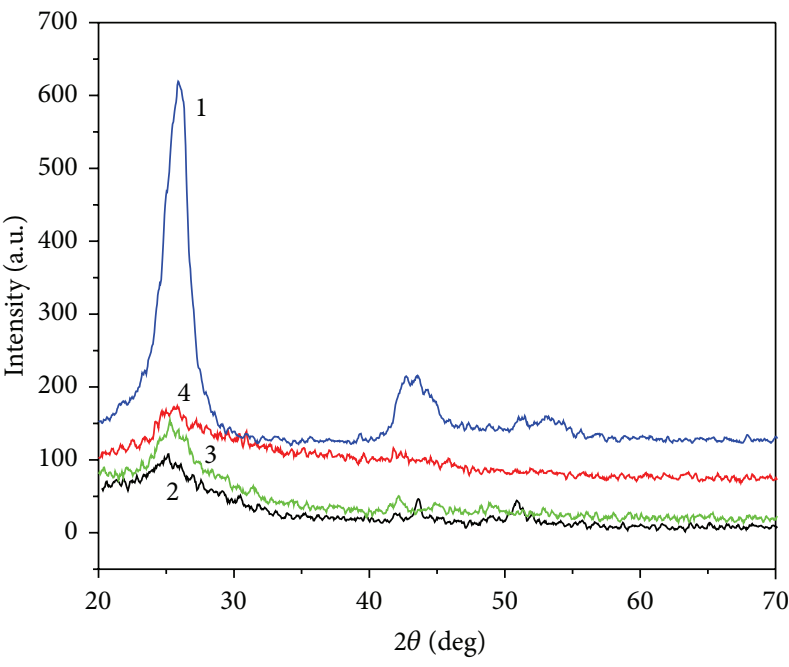

(1) SWCNT

(2) SPANI
(3) SPANI with $6 \%$ SWCNT

(4) SPANI with SWCNT/ZnS

(a)

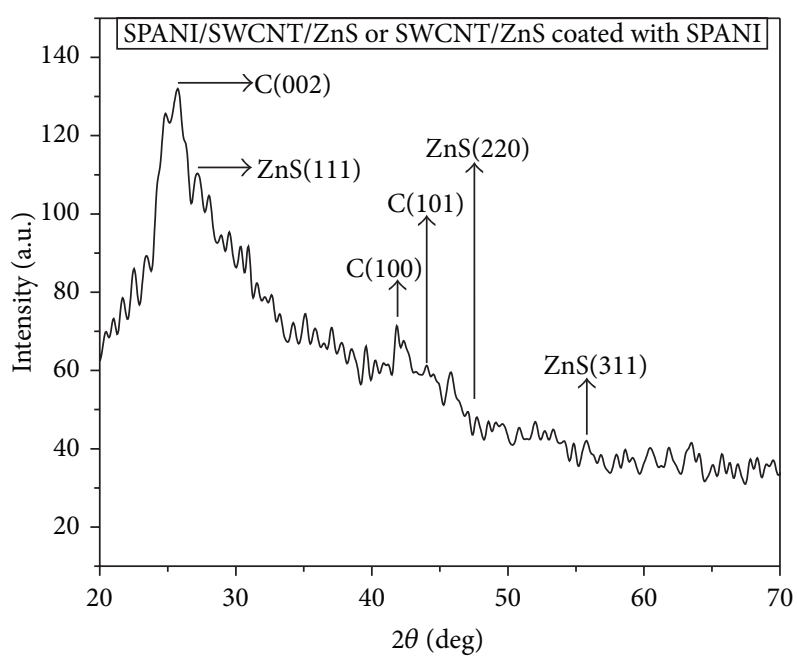

(b)

FIGURE 2: (a) XRD pattern of SWCNT, SPANI, SPANI/SWCNT, and SPANI/SWCNT/ZnS. (b) Smoothened XRD pattern of SPANI/SWCNT/ $\mathrm{ZnS}$ structure.

which are embedded in the matrix of sulfonated polyaniline. The average size of the zinc sulfide nanoparticles is found to be $10 \mathrm{~nm}$ by direct measurement in the micrograph.

3.1.2. XRD Pattern. The structural characteristics of SPANI, SWCNT, SPANI/SWCNT, and SPANI/SWCNT/ZnS have been analyzed by X-ray diffractograms shown in Figures 2(a) and 2(b). In the XRD pattern of SPANI, there are three prominent humps at $2 \theta=25^{\circ}, 43.5^{\circ}$ and $51^{\circ}$. No crystalline peak of SPANI was observed as SPANI is amorphous. In the pattern of SWCNT, the peaks at $26^{\circ}, 42^{\circ}$, and $44^{\circ}$ correspond to (002), (100), and (101) reflections of the graphitic planes of the nanotubes, respectively (JCPDS card no. 75-1621). In the XRD pattern of SPANI-SWCNT composite, no new peak appeared. In the XRD pattern of SPANI/SWCNT/ZnS, apart from the humps assigned to SPANI and peaks assigned to SWCNT, we also observe the peaks assigned to (111), (220), and (311) planes of the cubic phase of $\mathrm{ZnS}$ crystal structure (JCPDS card no. 05-0566).

The mean size $(D)$ of $\mathrm{ZnS}$ nanoparticles of the composite system was calculated using Debye-Scherrer formula:

$$
D=\frac{K \lambda}{\beta \cos \theta},
$$

where $K=0.89, \lambda$ is the wavelength of $\mathrm{Cu} K_{\alpha}$ radiation, $\beta$ is the full width at half maximum (FWHM) of the diffraction peak, and $\theta$ is the diffraction angle. The peak assigned to plane (111) of $\mathrm{ZnS}$ is chosen to calculate the particle size.
The average size of the $\mathrm{ZnS}$ nanocrystallite is estimated to be around $2 \mathrm{~nm}$. The disparity in particle size between this calculation and micrograph measurement is due to the fact that the micrograph shows the aggregates of smaller particles in the matrix of SPANI.

3.1.3. FTIR Spectra. In FTIR spectra as shown in Figure 3, the stretches at different points indicate the absorption bands. The stretch near $3450 \mathrm{~cm}^{-1}$ indicates the stretching vibration arising from the absorption of water by $\mathrm{KBr}$ used in the analysis. The spectrum of pristine SWCNT is almost featureless. In SWCNT/ZnS hybrid, the stretches at 1110 and $1383 \mathrm{~cm}^{-1}$ arise due to the presence of sulphur compound bondings, which were absent in pristine SWCNT. The stretch at $1630 \mathrm{~cm}^{-1}$ is due to the deformation vibration originated from absorption of water by $\mathrm{KBr}$ matrix [13].

Pure PANI shows the characteristic stretches at 1560 , 1480,1300 , and $1140 \mathrm{~cm}^{-1}$. The stretches at 1560 and $1480 \mathrm{~cm}^{-1}$ arise due to the stretching vibrations of quinoid ring $(-\mathrm{N}=$ quinoid $=\mathrm{N}-)$ and the benzenoid ring $(-\mathrm{N}$-benzenoid$\mathrm{N}-$ ), respectively. The stretch at $1300 \mathrm{~cm}^{-1}$ is due to $\mathrm{C}-\mathrm{N}$ stretching and that at $1140 \mathrm{~cm}^{-1}$ is due to $\mathrm{C}=\mathrm{N}$ stretching. In SPANI, the stretches at 700 and $615 \mathrm{~cm}^{-1}$ arise due to S$\mathrm{O}$ and $\mathrm{C}-\mathrm{S}$ stretching vibrations, respectively. The stretch at $815 \mathrm{~cm}^{-1}$ is assigned to the aromatic C-H out-of-plane bending vibration of the 1,2,4-trisubstituted aromatic rings. Similar results have been reported earlier [14]. This suggests that $-\mathrm{SO}_{3} \mathrm{H}$ groups were directly attached to the aromatic rings. The FTIR spectra of SPANI/SWCNT/ZnS composite 


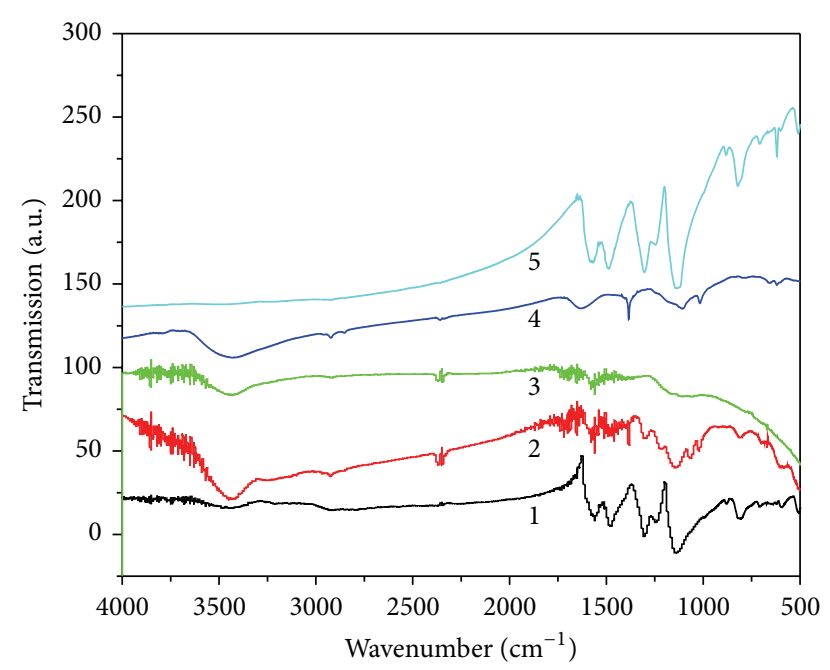
(1) PANI
(4) SWCNT/ZnS nanohybrid
(2) SPANI
(3) SWCNT
(5) SPANI/SWCNT/ZnS nanocomposite

FIGURE 3: FTIR spectra of SPANI, SWCNT, SWCNT/ZnS, and SPANI/SWCNT/ZnS.

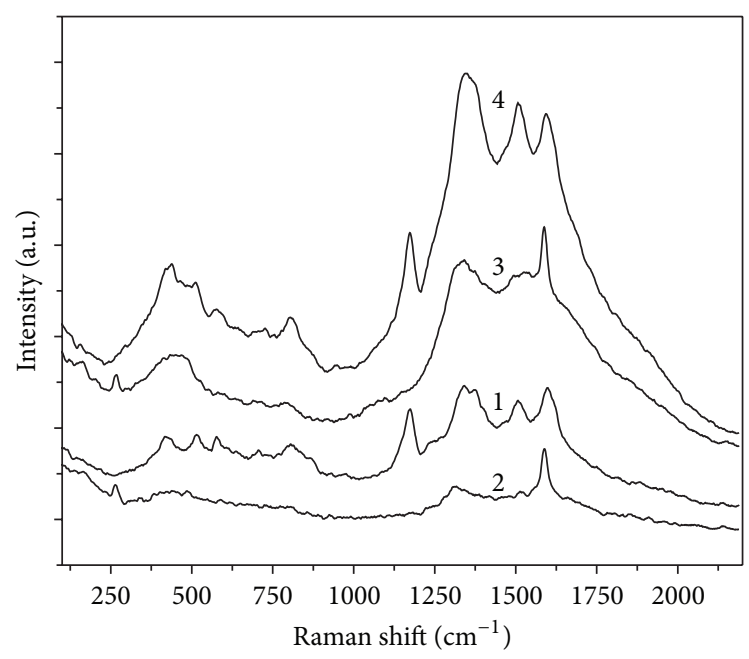
(1) SPANI
(3) SWCNT-ZnS
(2) SWCNT
(4) SPANI-SWCNT-ZnS

FIGURE 4: Raman spectra of SPANI, SWCNT, SWCNT/ZnS, and SPANI/SWCNT/ZnS.

almost match with those of SPANI. This indicates that the surface of SWCNT/ZnS hybrid was coated with SPANI to form SPANI/SWCNT/ZnS composite. The FTIR spectrum confirms that the SWCNT/ZnS hybrid fibers are embedded in the polymer matrix.

3.1.4. Raman Spectra. The Raman spectrum of SWCNT in Figure 4 shows the normal characteristic peaks, the G-band at $1589 \mathrm{~cm}^{-1}$, the D-band at $1314 \mathrm{~cm}^{-1}$, and the radial breathing mode (RBM) band at $265 \mathrm{~cm}^{-1}$. There is no appreciable shift of these bands for SWCNT/ZnS hybrid nanostructure, as reported earlier [13]. In case of SPANI, the peak at $1596 \mathrm{~cm}^{-1}$

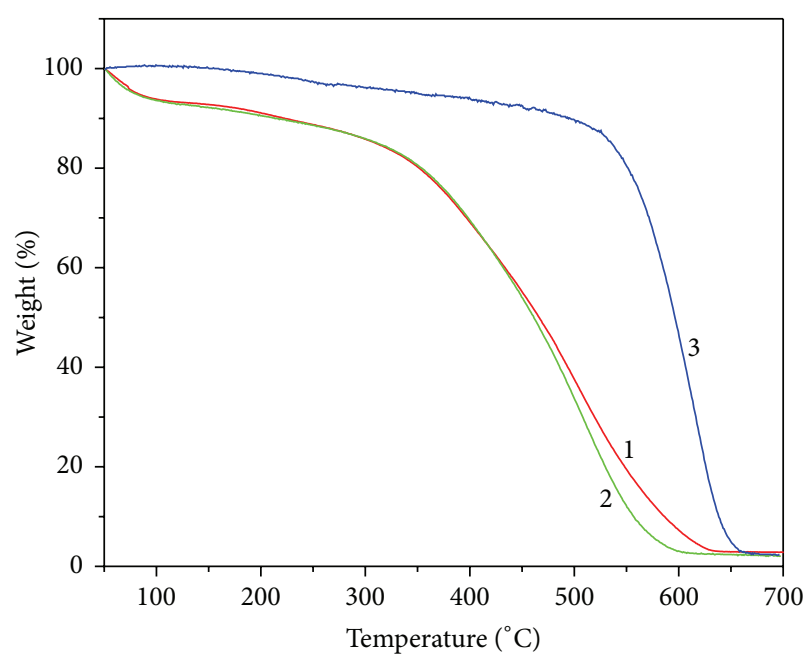

(1) SPANI with $6 \%$ SWCNT

(2) SPANI with SWCNT/ZnS

(3) SWCNT

FIGURE 5: TGA thermograms of SPANI, SPANI/SWCNT/ZnS, and SWCNT.

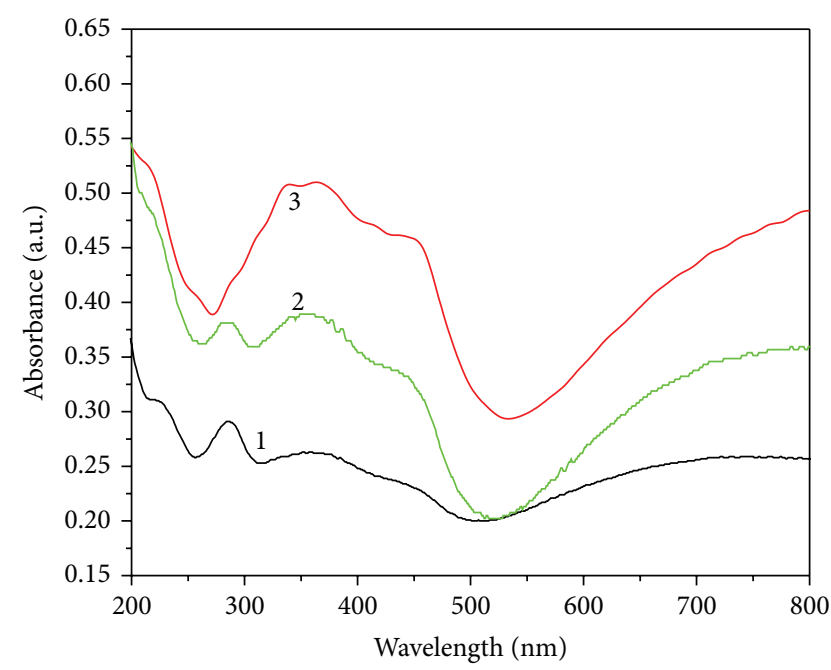

(1) SPANI

(2) SPANI with $6 \%$ SWCNT

(3) SPANI with SWCNT/ZnS

FIGURE 6: UV-vis absorbance spectra of SPANI, SPANI/SWCNT, and SPANI/SWCNT/ZnS.

arises from $\mathrm{C}-\mathrm{C}$ stretching of the benzenoid ring. The peak at $1504 \mathrm{~cm}^{-1}$ comes from $\mathrm{C}=\mathrm{N}$ stretching of the quinoid ring. The peak at 1340 arises from $\mathrm{C}-\mathrm{N}$ stretching and that at 1170 arises from $\mathrm{C}-\mathrm{H}$ bending of the quinoid/benzenoid ring. The $I_{\mathrm{D}} / I_{\mathrm{G}}$ factor (ratio between the intensity of $\mathrm{D}$ band and that of $\mathrm{G}$ band) increased on coating SWCNT/ZnS hybrid with the polymer. For SWCNT/ZnS hybrid, the $I_{\mathrm{D}} / I_{\mathrm{G}}$ factor is 0.7644, while for SPANI/SWCNT/ZnS composite, it has been found to be 1.1176. This increase in $I_{\mathrm{D}} / I_{\mathrm{G}}$ factor indicates that the SPANI adhered to the hybrid nanostructure via chemical bonding. The RBM band observed at $265 \mathrm{~cm}^{-1}$ for SWCNT 

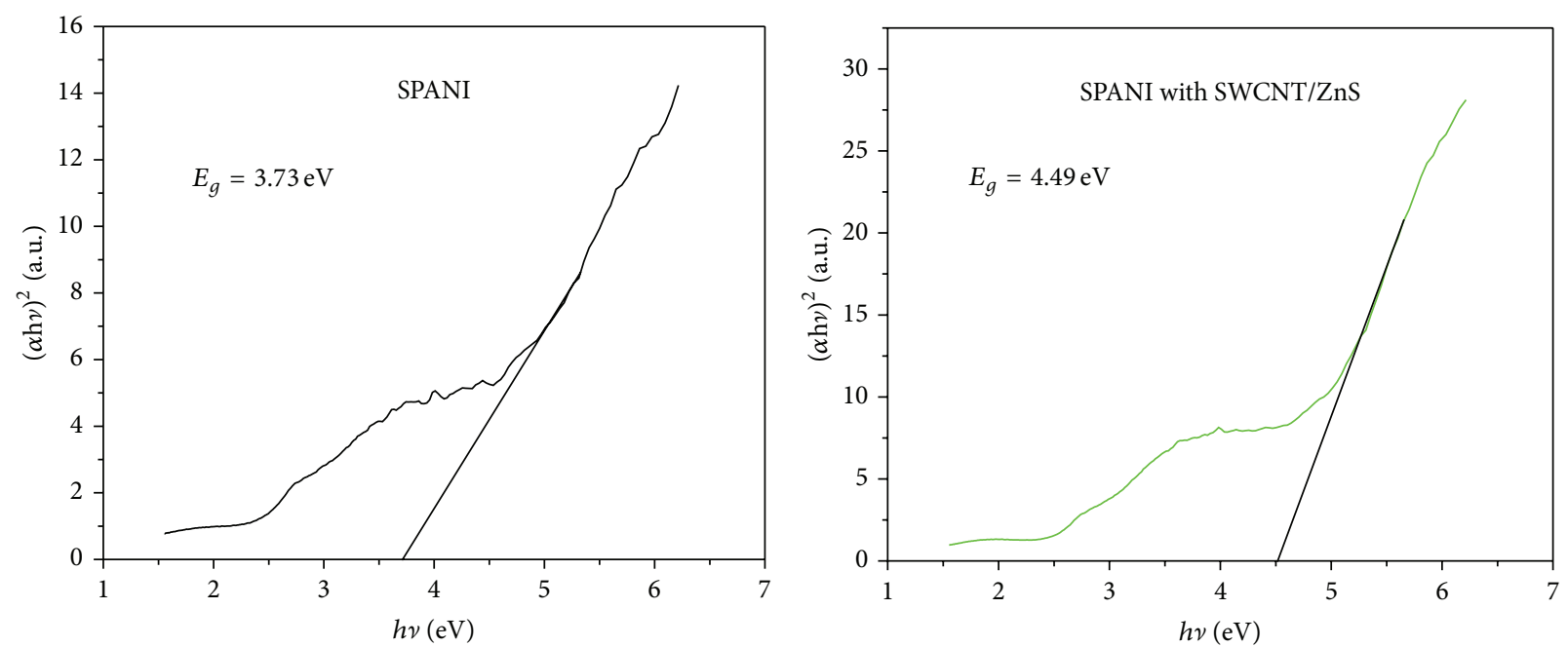

FIGURE 7: Plot of $(\alpha h \nu)^{2}$ versus $h \nu$ for SPANI and SPANI/SWCNT/ZnS composite.

has blue-shifted to $440 \mathrm{~cm}^{-1}$ for the composite material. Such blue shift can be attributed to the charge transfer between the polymer and the nanotube. After coating of the hybrid nanostructure with SPANI, the spectrum of the polymer dominates. This observation indicates that a good coating of the SWCNT/ZnS has been achieved.

3.1.5. TGA Study. The rogravimetric analysis (TGA) was done to investigate the thermal stability of the samples. Figure 5 shows the TGA thermograms of SWCNT, SPANI/ SWCNT and SPANI/SWCNT/ZnS composites. The SWCNT has a higher thermal stability than other samples and it shows no appreciable mass loss up to about $550^{\circ} \mathrm{C}$. The thermograms of SPANI/SWCNT, and SPANI/SWCNT/ZnS composites are similar up to $450^{\circ} \mathrm{C}$ but beyond $450^{\circ} \mathrm{C}$, the mass loss for the former is slightly lower than the latter, which indicates higher thermal stability for SPANI/SWCNT/ZnS composite. This is due to the presence of SWCNT/ZnS hybrid structures in the nanocomposite.

\subsection{Optical Characterization}

3.2.1. UV-Visible Absorption Spectra. The UV-visible absorption spectra of SPANI, SPANI/SWCNT, and SPANI/SWCNT/ $\mathrm{ZnS}$ are compared and shown in Figure 6. The spectrum of SPANI consists of a prominent peak at $285 \mathrm{~nm}$ and a hump around $370 \mathrm{~nm}$. The peak at $285 \mathrm{~nm}$ is assigned to the $\pi-\pi^{*}$ transition. The hump around $370 \mathrm{~nm}$ arises because of exciton transition on the polyemeraldine chain. A blue shift of the exciton transition from $370 \mathrm{~nm}$ (SPANI) to $360 \mathrm{~nm}$ (SPANI with $6 \%$ SWCNT) was observed due to the increase in the $\pi$-conjugation of the SPANI backbone. This demonstrates an electrostatic interaction between SPANI and SWCNT. The spectrum of SPANI/SWCNT/ZnS composite shows a broad maximum from $335 \mathrm{~nm}$ to $365 \mathrm{~nm}$. So there is a blue shift of the exciton transition band from $370 \mathrm{~nm}$ (SPANI) to an average of $350 \mathrm{~nm}$ (composite). This is due to synergetic effects of SPANI and SWCNT/ZnS hybrid by way of their electronic interaction and charge transfer.
The optical band gap $E_{g}$ is related to the absorption coefficient $\alpha$ by the relation

$$
\alpha=\frac{B\left(h v-E_{g}\right)^{1 / 2}}{h \nu},
$$

where $B$ is the absorption constant for a direct transition. We have plotted in Figure $7(\alpha h \nu)^{2}$ versus $h v$ and extrapolated the linear portion of it to $\alpha=0$ value and obtained the corresponding band gaps. The optical band gap of pure SPANI was estimated to be $3.73 \mathrm{eV}$. For SPANI/SWCNT/ZnS composite, there is an increase in the band gap energy, with the value being $4.49 \mathrm{eV}$. The estimated band gap of pure $\mathrm{ZnS}$ is $4.59 \mathrm{eV}$ [9].

3.2.2. Photoluminescence Spectra. Figure 8(a) shows the photoluminescence spectra of SPANI, SPANI/SWCNT and SPANI/SWCNT/ZnS composite obtained at an excitation wavelength of $300 \mathrm{~nm}$. SPANI shows a broad peak at $442 \mathrm{~nm}$. We observe a slight blue shift to $440 \mathrm{~nm}$ for SPANI with $6 \%$ SWCNT. The peak position is further blue-shifted to $416 \mathrm{~nm}$ for SPANI/SWCNT/ZnS composite with enhanced intensity. When the SPANI/SWCNT/ZnS composite is excited by a range of excitation wavelengths from 270 to $330 \mathrm{~nm}$, the emission band is gradually blue-shifted and with further increase in wavelength, we observe a slight red shift, with a prominent emission peak at $360 \mathrm{~nm}$ excitation wavelength as shown in Figure 8(b). When the SPANI/SWCNT/ZnS composite is excited, the electrons transit instantly from $\mathrm{ZnS}$ to SWCNT, causing emission with enhanced intensity. The change in band gap causes a blue shift.

\section{Conclusions}

We reported the synthesis of a novel luminescent material containing sulfonated polyaniline, embedded with functionalized SWCNTs hybridized with ZnS nanocrystals, by a simple chemical process. Characterization established the desired nanostructure of the composite material. The energy 


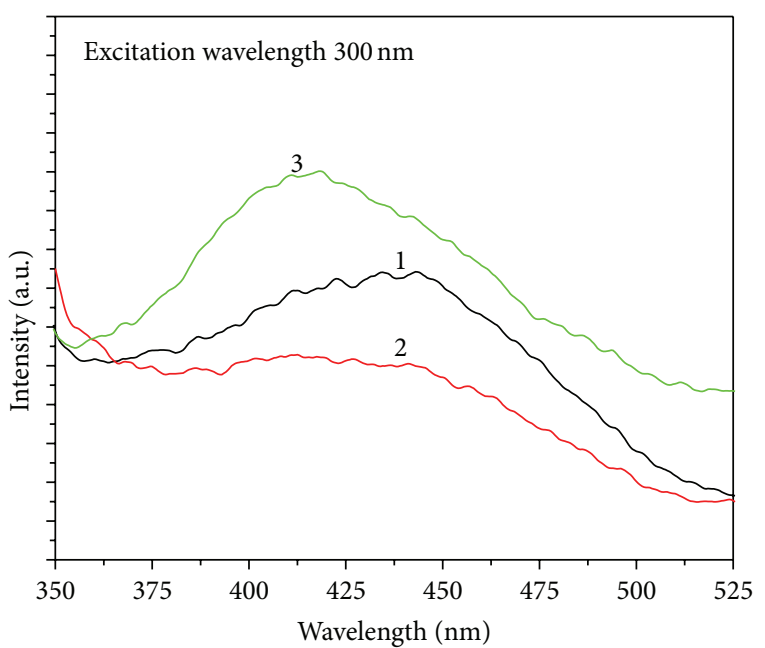

(1) SPANI

(2) SPANI with 6\% SWCNT

(3) SPANI with SWCNT/ZnS hybrid

(a)

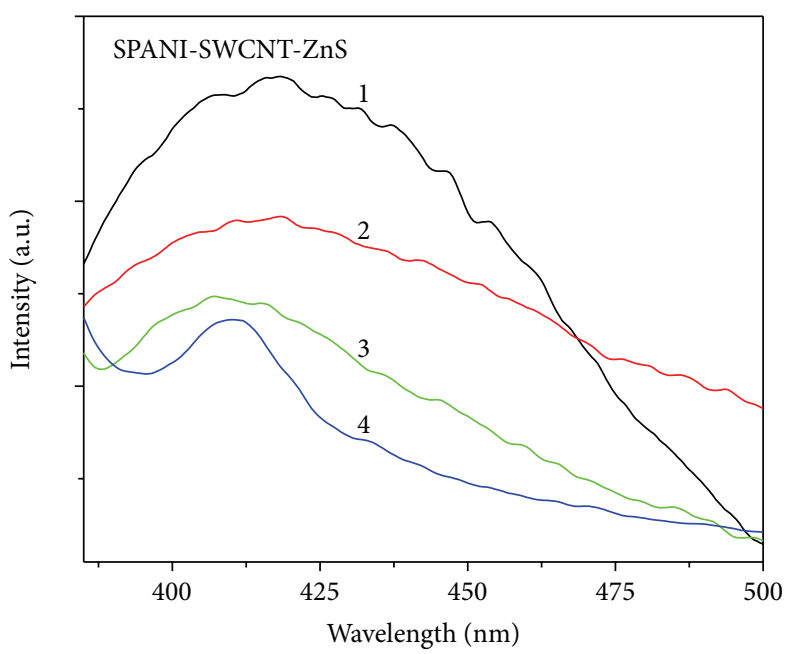

(1) Excitation wavelength $270 \mathrm{~nm}$ (3) Excitation wavelength $330 \mathrm{~nm}$

$\begin{array}{ll}\text { (2) Excitation wavelength } 300 \mathrm{~nm} & \text { (4) Excitation wavelength } 360 \mathrm{~nm}\end{array}$

(b)

FIGURE 8: (a) Photoluminescence spectra for SPANI, SPANI/ SWCNT, and SPANI/SWCNT/ZnS. (b) Photoluminescence spectra for SPANI/SWCNT/ZnS nanocomposite at different excitation wavelengths.

band gap of $3.73 \mathrm{eV}$ for pure SPANI was found to be increased to $4.49 \mathrm{eV}$ for the composite. The PL emission from the composite showed a broad emission range in the visible region between 400 and $450 \mathrm{~nm}$ with the peak emission at $416 \mathrm{~nm}$, with appreciable enhancement in the intensity of emission, leading to the formation of a novel luminescent polymer nanocomposite.

\section{References}

[1] S. Iijima, "Helical microtubules of graphitic carbon," Nature, vol. 354, no. 6348, pp. 56-58, 1991.
[2] M. S. Dresselhaus and H. Dai, "Carbon nanotubes: continued innovations and challenges," MRS Bulletin, vol. 29, no. 4, pp. 237-243, 2004.

[3] C. Dhand, P. R. Solanki, M. Datta, and B. D. Malhotra, "Polyaniline/single-walled carbon nanotubes composite based triglyceride biosensor," Electroanalysis, vol. 22, no. 22, pp. 2683-2693, 2010.

[4] V. Gupta and N. Miura, "Polyaniline/single-wall carbon nanotube (PANI/SWCNT) composites for high performance supercapacitors," Electrochimica Acta, vol. 52, no. 4, pp. 1721-1726, 2006.

[5] P. C. Ramamurthy, A. M. Malshe, W. R. Harrell, R. V. Gregory, K. McGuire, and A. M. Rao, "Polyaniline/single-walled carbon nanotube composite electronic devices," Solid-State Electronics, vol. 48, no. 10-11, pp. 2019-2024, 2004.

[6] M. Tahhan, V.-T. Truong, G. M. Spinks, and G. G. Wallace, "Carbon nanotube and polyaniline composite actuators," Smart Materials and Structures, vol. 12, no. 4, pp. 626-632, 2003.

[7] W. Tang and D. C. Cameron, "Electroluminescent zinc sulphide devices produced by sol-gel processing," Thin Solid Films, vol. 280, no. 1-2, pp. 221-226, 1996.

[8] T. V. Prevenslik, "Acoustoluminescence and sonoluminescence," Journal of Luminescence, vol. 87, pp. 1210-1212, 2000.

[9] K. Dutta, S. Manna, and S. K. De, "Optical and electrical characterizations of $\mathrm{ZnS}$ nanoparticles embedded in conducting polymer," Synthetic Metals, vol. 159, no. 3-4, pp. 315-319, 2009.

[10] H. C. Pant, M. K. Patra, S. C. Negi, A. Bhatia, S. R. Vadera, and N. Kumar, "Studies on conductivity and dielectric properties of polyaniline-zinc sulphide composites," Bulletin of Materials Science, vol. 29, no. 4, pp. 379-384, 2006.

[11] S. D. Bompilwar, S. B. Kondawar, V. A. Tabhane, and S. R. Kargirwar, "Thermal stability of CdS/ZnS nanoparticles embedded conducting polyaniline nanocomposites," Advances in Applied Science Research, vol. 1, no. 1, p. 166, 2010.

[12] A. Singh, N. P. Singh, P. Singh, and R. A. Singh, "Synthesis and characterization of conducting polymer composites based on polyaniline-polyethylene glycol-zinc sulfide system," Journal of Polymer Research, vol. 18, no. 1, pp. 67-77, 2011.

[13] R. Paul, P. Kumbhakar, and A. K. Mitra, "Synthesis and study of photoluminescence characteristics of carbon nanotube/ZnS hybrid nanostructures," Journal of Experimental Nanoscience, vol. 5, no. 4, pp. 363-373, 2010.

[14] Y.-W. Lin and T.-M. Wu, "Synthesis and characterization of externally doped sulfonated polyaniline/multi-walled carbon nanotube composites," Composites Science and Technology, vol. 69, no. 15-16, pp. 2559-2565, 2009.

[15] R. Sainz, A. M. Benito, M. T. Martínez et al., "A soluble and highly functional polyaniline-carbon nanotube composite," Nanotechnology, vol. 16, no. 5, pp. S150-S154, 2005.

[16] M. Scocioreanu, M. Baibarac, I. Baltog, I. Pasuk, and T. Velula, "Photoluminescence and Raman evidence for mechanicochemical interaction of polyaniline-emeraldine base with $\mathrm{ZnS}$ in cubic and hexagonal phase," Journal of Solid State Chemistry, vol. 186, pp. 217-223, 2012. 

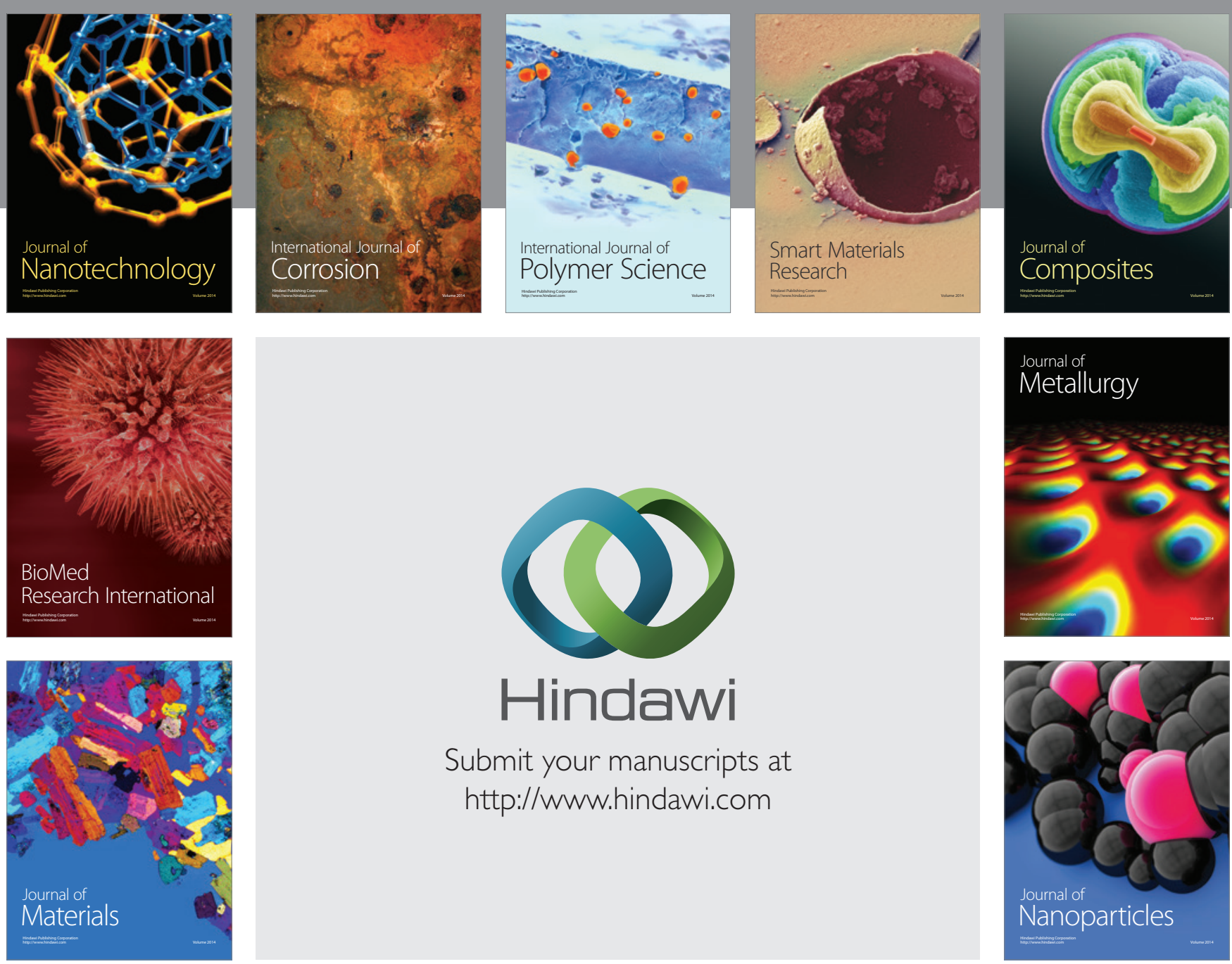

Submit your manuscripts at http://www.hindawi.com
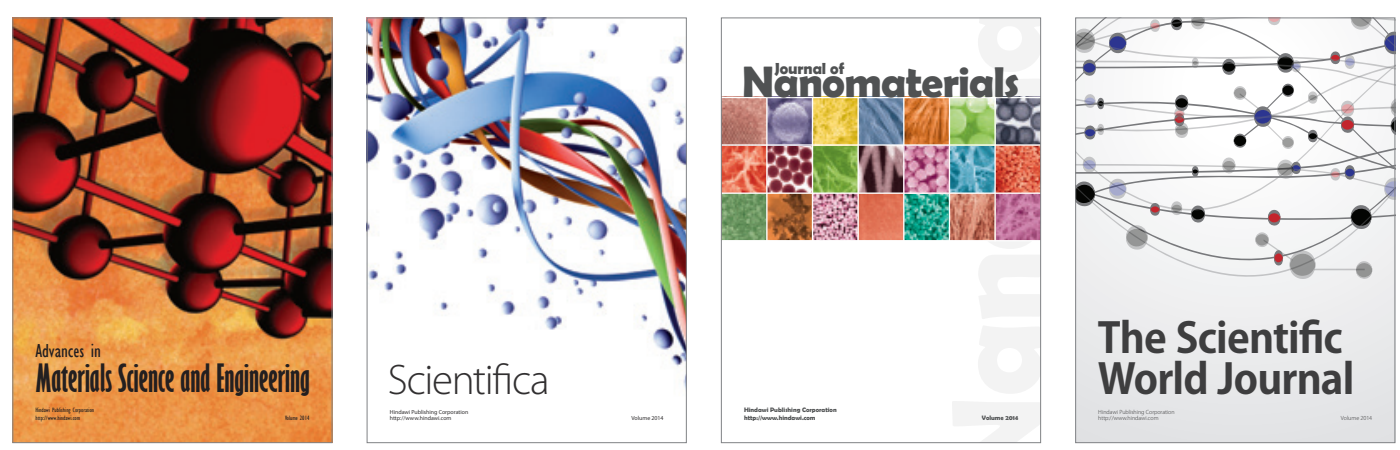

\section{The Scientific World Journal}
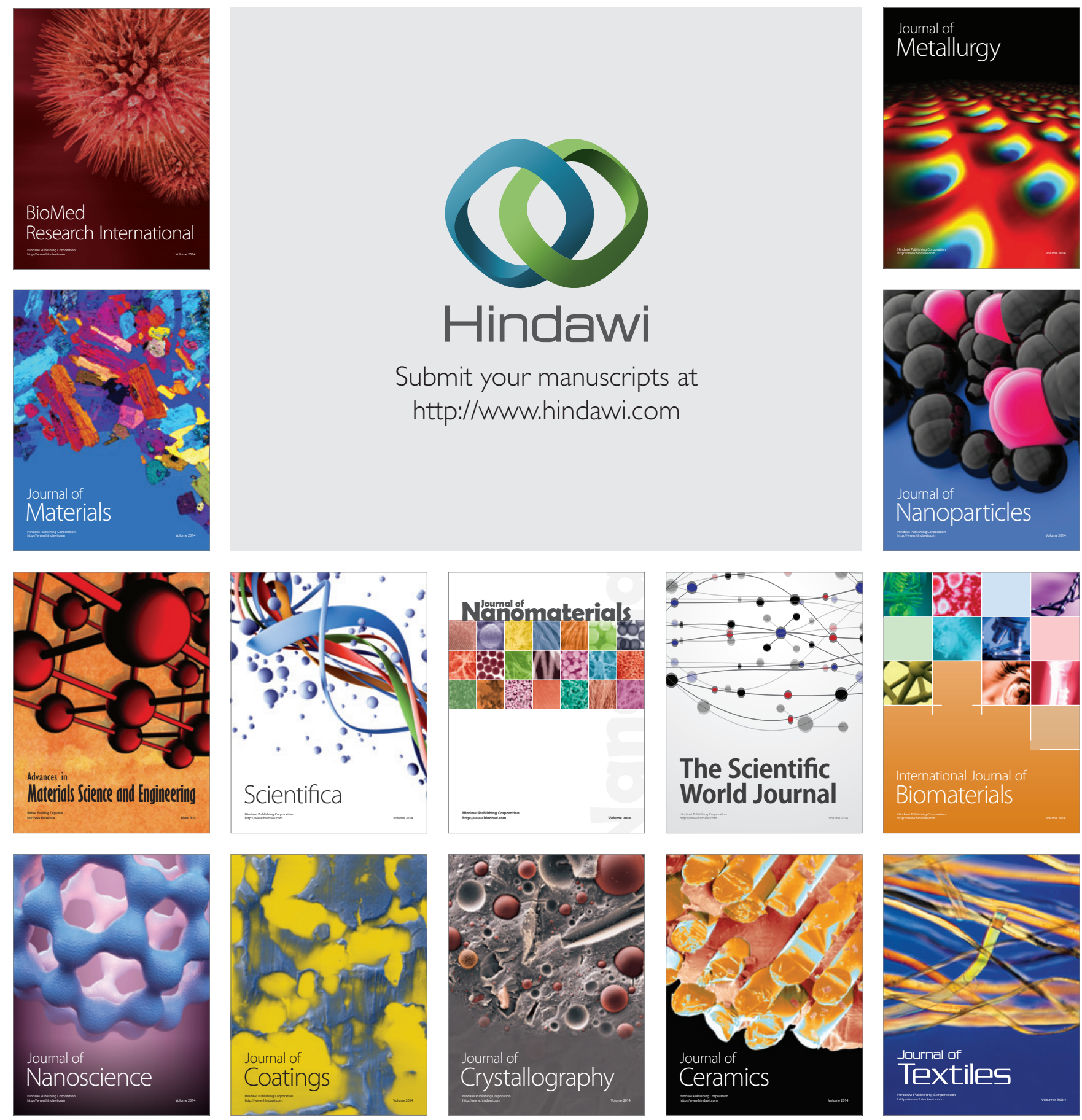